\title{
Entropy exchange, coherent information and concurrence
}

\author{
Yang Xiang* and Shi-Jie Xiong \\ National Laboratory of Solid State Microstructures and Department of Physics, Nanjing University, Nanjing 210093, China
}

(Dated: August 23, 2021)

\begin{abstract}
For a simple model we derive analytic expressions of entropy exchange and coherent information, from which relations between them and the concurrence are drawn. We find that in the quantum evolution the entropy exchange exhibits behavior opposite to that of the concurrence, whereas the coherent information shows features very similar to those of the concurrence. The meaning of this result for general systems is discussed.
\end{abstract}

PACS numbers: 03.67.Mn, 03.65.Ud

Quantum entanglement is not only of interest in the interpretation of the foundation of quantum mechanics, but also a resource useful in quantum information processing and quantum computation. A measure of the entanglement in a state of two qubits is the Wooters concurrence [1]. For pure bipartite states one also uses the Von Neumann entropy of the reduced density matrix for either of the parties [2, 3] to measure the entanglement. For a simple example, which has been proposed by Jordan et al. [4], we have shown that in the quantum evolution a modified entanglement fidelity exhibits the behavior similar to that of the concurrence [5]. In this paper we investigate other two quantities, the entropy exchange and the coherent information [6, 7], by deriving analytic expressions for the system proposed by Jordan et al.. From these we obtain relations between them and the concurrence in the quantum evolution and discuss the possibility of using them as measures of the entanglement.

We begin with a brief discussion of noisy quantum processes and their mathematical descriptions. $R$ and $Q$ are two quantum systems and the joint system $R Q$ is initially prepared in a pure entangled state $\left|\Psi^{R Q}\right\rangle$. The system $R$ is dynamically isolated and has a zero internal Hamiltonian, while the system $Q$ undergoes some evolution that possibly involves interaction with the environment. The evolution of $Q$ might represent a transmission process via some quantum channel for the quantum information in $Q$. In general, the evolution of $Q$ can be represented by a quantum operator $\varepsilon^{Q}$, which gives the mapping from the initial state to the final state

$$
\rho^{Q^{\prime}}=\varepsilon^{Q}\left(\rho^{Q}\right) .
$$

Here, $\rho^{Q}=\operatorname{Tr}_{R}\left|\Psi^{R Q}\right\rangle\left\langle\Psi^{R Q}\right|$ which represents the initial state of system $Q$, and after the dynamical process the final state of the system becomes $\rho^{Q^{\prime}}$. In the most general case, the map $\varepsilon^{Q}$ must be trace preserving and is a linear positive map [8, 9], so it can represent all unitary evolutions. The evolution may also include unitary evolving interactions with an environment $E$. Suppose that the environment is initially in state $\rho^{E}$. The operator can be

*Electronic address: njuxy@sina.com written as

$$
\begin{aligned}
\varepsilon^{Q}\left(\rho^{Q}\right) & =\operatorname{Tr}_{E} U\left(\rho^{Q} \otimes \rho^{E}\right) U^{\dagger} \\
& =\operatorname{Tr}_{E} U\left(\rho^{Q} \otimes \sum_{i} p_{i}|i\rangle\langle i|\right) U^{\dagger} \\
& =\sum_{j} E_{j}^{Q} \rho^{Q} E_{j}^{Q \dagger}
\end{aligned}
$$

where $\sum_{i} p_{i}|i\rangle\langle i|$ is the spectral decomposition of $\rho^{E}$ with $\{|i\rangle\}$ being a base in the Hilbert space $\mathcal{H}_{E}$ of the environment $E$, and $E_{j}^{Q}=\sum_{i} \sqrt{p_{i}}\langle j|U| i\rangle$. The final state of $R Q$ is

$$
\begin{aligned}
\rho^{R Q^{\prime}} & =\mathcal{I}^{R} \otimes \varepsilon^{Q}\left(\rho^{R Q}\right) \\
& =\sum_{j}\left(1^{R} \otimes E_{j}^{Q}\right) \rho^{R Q}\left(1^{R} \otimes E_{j}^{Q}\right)^{\dagger} .
\end{aligned}
$$

The entropy exchange $S_{e}$ is defined as [7]

$$
S_{e}=-\operatorname{Tr} \rho^{R Q^{\prime}} \log \rho^{R Q^{\prime}} .
$$

It is the von Neumann entropy of the final state of the joint system $R Q$. Throughout this paper we use log to denote $\log _{2}$. The intrinsic expression of the entropy exchange is given by $S_{e}=S(W), S(\rho)$ is the von Neumann entropy of density operator $\rho$ and $W$ is a density operator with components (in an orthonormal basis)

$$
W_{i j}=\operatorname{Tr} E_{i}^{Q} \rho^{Q} E_{j}^{Q \dagger} .
$$

The entropy exchange is an intrinsic property of $Q$, depending only on $\rho^{Q}$ and $\varepsilon^{Q}$. It is a measure of the information exchanged between system $Q$ and the environment during evolution $\varepsilon^{Q}$. If there is no interaction between system $Q$ and the environment, i.e., $\varepsilon^{Q}$ is a unitary operator, then after the dynamical process the final state of the joint system $R Q$ is still a pure state. This means that in this case the entropy exchange equals zero. A complete discussion of the entropy exchange can be seen in Refs. [6, 7, [10]. In Ref. [5] we show that the modified entanglement fidelity (MEF) admirably reflects the entanglement preservation. The MEF is defined as

$$
F_{e}=\max _{U Q}\left\langle\Psi^{R Q}\left|\left(1^{R} \otimes U^{Q}\right) \rho^{R Q^{\prime}}\left(1^{R} \otimes U^{Q}\right)^{\dagger}\right| \Psi^{R Q}\right\rangle,
$$


where $U^{Q}$ is a unitary transformation acting on $Q$. A connection between the MEF and the entropy exchange is given by the quantum Fano inequality [7],

$$
h\left(F_{e}\right)+\left(1-F_{e}\right) \log \left(d^{2}-1\right) \geq S_{e},
$$

where $h(\rho)=-\rho \log \rho-(1-\rho) \log (1-\rho)$ and $d$ is the number of the complex dimensions of the Hilbert space $\mathcal{H}$ describing system $Q$. We can easily find that when $F_{e}=1$, i.e., the entanglement is admirably preserved in the evolution process, $S_{e}$ equals zero. This implies that there may be some connection between the entropy exchange and the measures of the entanglement, e.g., the concurrence.

Another important intrinsic quantity is the coherent information $I_{e}$. It is defined as [6]

$$
I_{e}=S\left(\rho^{Q^{\prime}}\right)-S\left(\rho^{R Q^{\prime}}\right)=S\left(\rho^{Q^{\prime}}\right)-S_{e}
$$

For classical systems, $I_{e}$ can never be positive since the entropy of the joint system $R Q$ can never be less than the entropy of the subsystem $Q$. But, for quantum systems, $I_{e}$ may take positive value. For example, if $\rho^{R Q^{\prime}}$ is an entangled pure state, then $\rho^{Q^{\prime}}$ is a mixed state, implying that $S\left(\rho^{Q^{\prime}}\right)>0$ and $S\left(\rho^{R Q^{\prime}}\right)=0$. Thus, $I_{e}$ can be regarded as a measure of the "nonclassicity" of the final joint state $\rho^{R Q^{\prime}}$, or, in other words, the degree of the quantum entanglement retained by $R$ and $Q$.

Now we discuss a simple example introduced by Jordan et al. in their work [4]. For this example we can give analytic expressions of the entropy exchange and the coherent information from which the relations between them and the concurrence can be obtained. We consider two entangled qubits, $A$ and $B$, and suppose that qubit $A$ interacts with a control qubit $C$. Then $A, B$ and $C$ respectively correspond to systems $Q, R$ and environment $E$ discussed above. We suppose that the initial states of the three qubits are

$$
\Lambda=\rho^{A B} \otimes \frac{1}{2} 1_{c}
$$

where $\rho^{A B}$ is the initial state of joint system $A$ and $B$. According to the Schmidt theorem [11], a pure state of two $\frac{1}{2}$-spins can be decomposed as

$$
\begin{aligned}
|\Psi\rangle= & e^{-i \varphi / 2} \cos \left(\frac{\theta}{2}\right)|\mathbf{n}\rangle_{A}|\mathbf{m}\rangle_{B} \\
& +e^{i \varphi / 2} \sin \left(\frac{\theta}{2}\right)|-\mathbf{n}\rangle_{A}|-\mathbf{m}\rangle_{B}
\end{aligned}
$$

where $\mathbf{n}$ and $\mathbf{m}$ are two points on the Poincaré sphere, and the subscript specifies the related qubit $A$ or $B$. The "angle" $\theta$ in Eq. (10) determines the degree of entanglement in the state. The angle satisfies $0 \leq \theta \leq \pi$, $\theta=0$ and $\theta=\pi$ correspond to the product states, and the maximal entanglement is obtained for $\theta=\frac{\pi}{2}$. Without losing generality we set the initial state of the joint system $A$ and $B$ as

$$
|\psi\rangle=\cos \left(\frac{\theta}{2}\right)|+z\rangle_{A}|-z\rangle_{B}+\sin \left(\frac{\theta}{2}\right)|-z\rangle_{A}|+z\rangle_{B}
$$

where $| \pm z\rangle$ represent the eigenstates of $\sigma_{z}$ with eigenvalues \pm 1 . So the density matrix $\rho^{A B}=|\psi\rangle\langle\psi|$. For simplicity, we omit the "azimuthal angle" $\varphi$, as factor $e^{ \pm i \varphi / 2}$ can be absorbed in eigenstates $| \pm z\rangle$ and has no effect on the final results.

We suggest an interaction between qubits $A$ and $C$ described by the unitary transformation

$$
U=e^{-i t H}
$$

where

$$
H=\frac{\lambda \sigma_{z}^{A}}{2}(|\alpha\rangle\langle\alpha|-| \beta\rangle\langle\beta|)
$$

with $\lambda$ being the strength of the interaction, and $|\alpha\rangle$ and $|\beta\rangle$ are two orthonormal vectors for system $C$. Then the changing density matrix for joint system of $A$ and $B$ can be calculated as

$$
\begin{aligned}
\rho^{A B^{\prime}} & =\operatorname{Tr}_{c}\left[\left(U \otimes 1^{B}\right) \Lambda\left(U \otimes 1^{B}\right)^{\dagger}\right]=\operatorname{Tr}_{c}\left[\left(U \otimes 1^{B}\right)\left(|\psi\rangle\langle\psi| \otimes \frac{1}{2} 1_{c}\right)\left(U \otimes 1^{B}\right)^{\dagger}\right] \\
& =\left(\begin{array}{llll}
0 & 0 & 0 & 0 \\
0 & \cos ^{2}\left(\frac{\theta}{2}\right) & \cos (\lambda t) \cos \left(\frac{\theta}{2}\right) \sin \left(\frac{\theta}{2}\right) & 0 \\
0 & \cos (\lambda t) \cos \left(\frac{\theta}{2}\right) \sin \left(\frac{\theta}{2}\right) & \sin ^{2}\left(\frac{\theta}{2}\right) & 0 \\
0 & 0 & 0 & 0
\end{array}\right) .
\end{aligned}
$$

The changing density matrix $\rho^{A B^{\prime}}$ usually represents a mixed state. In order to quantify the entanglement we adopt the Wootters concurrence [1] defined as

$$
C(\rho) \equiv \max \left[0, \sqrt{\lambda_{1}}-\sqrt{\lambda_{2}}-\sqrt{\lambda_{3}}-\sqrt{\lambda_{4}}\right]
$$

where $\rho$ is the density matrix representing the investigated state of the joint system of $A$ and $B, \lambda_{1}, \lambda_{2}, \lambda_{3}$, and $\lambda_{4}$ are the eigenvalues of $\rho \sigma_{2}^{A} \sigma_{2}^{B} \rho^{*} \sigma_{2}^{A} \sigma_{2}^{B}$ in the decreasing order, and $\rho^{*}$ is the complex conjugation of $\rho$. 
From Eq. (14) we can obtain

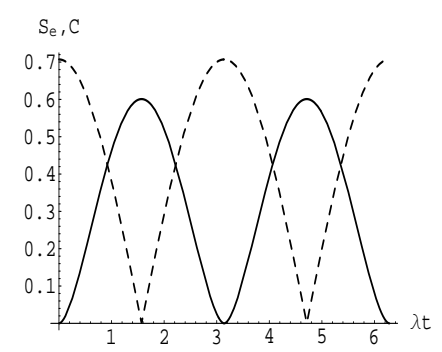

(a) $\theta=\pi / 4$

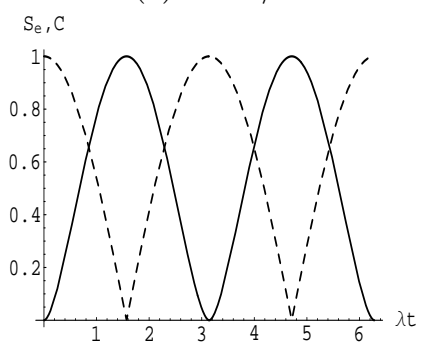

(c) $\theta=\pi / 2$

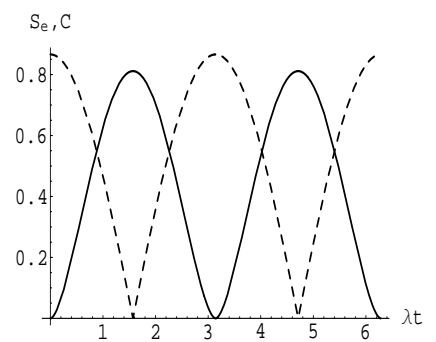

(b) $\theta=\pi / 3$

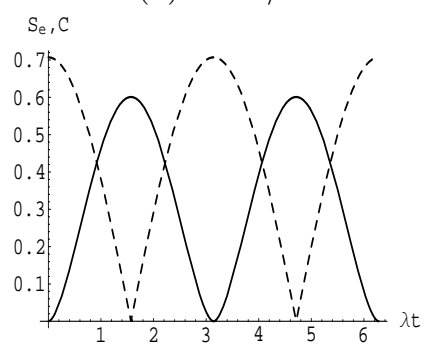

(d) $\theta=3 \pi / 4$

FIG. 1: Evolutions of the entropy exchange $S_{e}$ (solid line) and the concurrence $C$ (dashed line). We take $\hbar=1$ so that $\lambda t$ is dimensionless.

$$
C\left(\rho^{A B^{\prime}}\right)=\sin \theta|\cos \lambda t|
$$

It is found that at time $\lambda t=\frac{\pi}{2}$, the state $\rho^{A B^{\prime}}$ is changed from the initial entangled state at $t=0$ to a separable state, while at time $\lambda t=\pi$ the state $\rho^{A B^{\prime}}$ returns to the state with the initial entanglement. We can also find that if the initial state is a product state, i.e., $\theta=0$ or $\theta=\pi$, the concurrence always equals zero.

Using Eqs. (22), (9), (12), and (13), we obtain the quantum operation on qubit $A$,

$$
\begin{aligned}
\varepsilon^{A}\left(\rho^{A}\right)= & \operatorname{Tr}_{C} U\left(\rho^{A} \otimes \rho^{C}\right) U^{\dagger} \\
= & \operatorname{Tr}_{C} U\left(\rho^{A} \otimes\left(\frac{1}{2}(|\alpha\rangle\langle\alpha|+| \beta\rangle\langle\beta|)\right)\right) U^{\dagger} \\
= & \frac{1}{2} e^{-i \sigma_{3}^{A}\left(\frac{\lambda t}{2}\right)} \rho^{A} e^{+i \sigma_{3}^{A}\left(\frac{\lambda t}{2}\right)} \\
& +\frac{1}{2} e^{+i \sigma_{3}^{A}\left(\frac{\lambda t}{2}\right)} \rho^{A} e^{-i \sigma_{3}^{A}\left(\frac{\lambda t}{2}\right)}
\end{aligned}
$$

So $E_{\alpha}^{A}=\frac{1}{\sqrt{2}} e^{-i \sigma_{3}^{A}\left(\frac{\lambda t}{2}\right)}$ and $E_{\beta}^{A}=\frac{1}{\sqrt{2}} e^{+i \sigma_{3}^{A}\left(\frac{\lambda t}{2}\right)}$. Substituting them into Eq. (5) and noting that $\rho^{A} \equiv$ $\operatorname{Tr}_{B}\left(\rho^{A B}\right)=\left(\begin{array}{ll}\cos ^{2}\left(\frac{\theta}{2}\right) & 0 \\ 0 & \sin ^{2}\left(\frac{\theta}{2}\right)\end{array}\right)$, we can get the density matrix $W$ in Eq. (5) as

$$
W=\frac{1}{2}\left(\begin{array}{ll}
1 & \cos (\lambda t)-i \sin (\lambda t) \cos (\theta) \\
\cos (\lambda t)+i \sin (\lambda t) \cos (\theta) & 1
\end{array}\right)
$$

Thus, we have

$$
\begin{aligned}
S_{e}= & -\frac{1}{2}\left[1-\sqrt{\cos ^{2}(\lambda t)+\cos ^{2}(\theta) \sin ^{2}(\lambda t)}\right] \log \left\{\frac{1}{2}\left[1-\sqrt{\cos ^{2}(\lambda t)+\cos ^{2}(\theta) \sin ^{2}(\lambda t)}\right]\right\} \\
& -\frac{1}{2}\left[1+\sqrt{\cos ^{2}(\lambda t)+\cos ^{2}(\theta) \sin ^{2}(\lambda t)}\right] \log \left\{\frac{1}{2}\left[1+\sqrt{\cos ^{2}(\lambda t)+\cos ^{2}(\theta) \sin ^{2}(\lambda t)}\right]\right\} .
\end{aligned}
$$

We can also directly calculate $S_{e}$ by using Eq. (14) and Eq. (4). From Eq. (19), we find that if the initial state is a product one, i.e., $\theta=0$ or $\theta=\pi$, the entropy exchange always equals zero.

The evolutions of the entropy exchange $S_{e}$ and the concurrence $C\left(\rho^{A B^{\prime}}\right)$ are depicted in Fig. 1. We can see that the entropy exchange exhibits the behavior opposite to that of the concurrence in the quantum evolution. During the evolution $\varepsilon^{A}$, the more information exchanged between $A$ and "environment" $C$, the more entanglement between $A$ and $B$ is lost.

Now we discuss the coherent information $I_{e}$. By using Eq. (14) we find that $\rho^{A^{\prime}}=\operatorname{Tr}_{B}\left(\rho^{A B^{\prime}}\right)=$ $\left(\begin{array}{ll}\cos ^{2}\left(\frac{\theta}{2}\right) & 0 \\ 0 & \sin ^{2}\left(\frac{\theta}{2}\right)\end{array}\right)$, so from Eq. (8) we can obtain $I_{e}$. The evolutions of the coherent information $I_{e}$ and the concurrence $C\left(\rho^{A B^{\prime}}\right)$ are depicted in Fig. 2, It can be seen that $I_{e}$ exhibits the behavior very similar to that of the concurrence in the quantum evolution, although their values are not exactly consistent with each other at all moments.

The negative correlation between the entropy exchange and the concurrence is a striking feature of the quantities. In this example the range of $S_{e}$ is in $(0,1)$, where the left-hand side of the Fano inequality (7) is a monotonic decreasing function of the MEF $F_{e}$. This means that for a quantum operation with a given $S_{e}$ there should be an upper bound of $F_{e}$. In Ref. [5] we have shown that $F_{e}$ exhibits the behavior similar to that of the concurrence in the quantum evolution. So for a quantum operation giving $S_{e}$ there is also an upper bound of the concurrence. This only gives a rough correlation between the concur- 


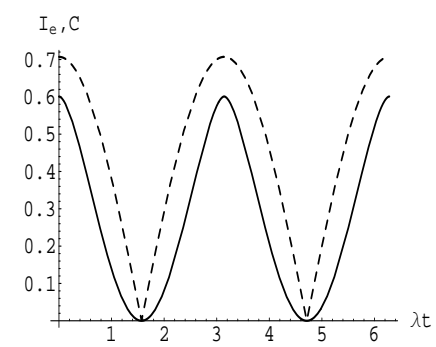

(a) $\theta=\pi / 4$

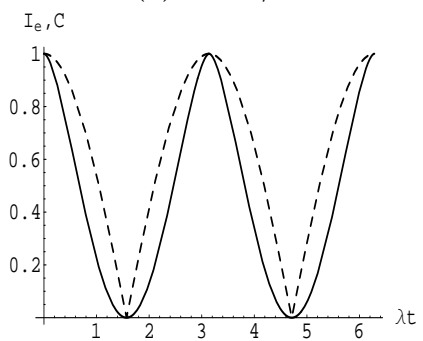

(c) $\theta=\pi / 2$

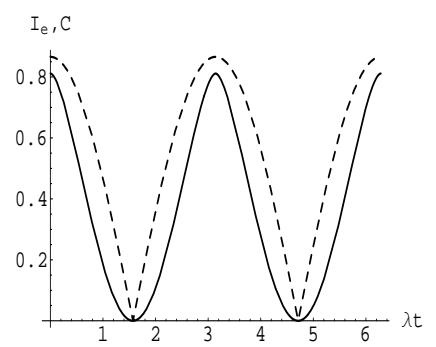

(b) $\theta=\pi / 3$

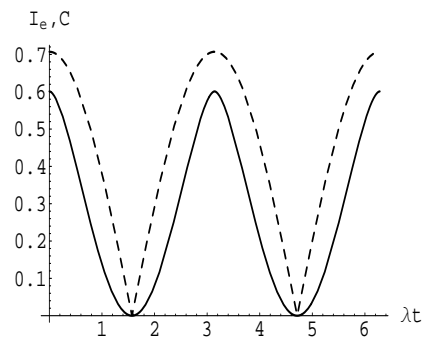

(d) $\theta=3 \pi / 4$
FIG. 2: Evolutions of the coherent information $I_{e}$ (solid line) and the concurrence $C$ (dashed line).

rence and the entropy exchange. Owing to the simplicity of the example we are able to obtain the analytical expressions of $F_{e}$ and $S_{e}$ and to illustrate the definite negative correlation between them. In this sample the effect of the environment is represented by only a single qubit $(C)$. In Eq. (9), we assumed the initial state of qubit $C$ is a mixed one. For a more complicated environment we may introduce an extra system, e.g., a qubit $D$, in addition to qubit $C$. If this new qubit purifies the initial state, then all the results obtained above are retained. In fact, we can regard the system $A B$ and the environment $C D$ as a new joint system and assume that the initial state of this joint system is a pure state. After a quantum operation which represents the interaction between subsystems $A$ and $C$, the final state of the joint system must be a pure state too, so the entropy exchange can also be seen as a measure of the entanglement between $A B$ and $C D$. The information exchange between $A$ and $C$, which is the result of the interaction, will cause the entanglement between $A B$ and $C D$, and decrease the entanglement between $A$ and $B$. This results in the opposite behavior between the entropy exchange and the concurrence in the quantum evolution. This also means that the entanglement between $A$ and $B$ and the entanglement between $A B$ and $C D$ have negative correlation. Thus, the conclusion about the negative correlation between the entropy exchange and the concurrence may be extended to the case of environment having two qubits but the initial state being purified. In other cases where the environment includes more qubits but there are no correlations between them, the effect of every qubit in the environment should be similar to that of the single qubit investigated in this example.

The coherent information $I_{e}$ depends only on $\rho^{Q}$ and $\varepsilon^{Q}$ and is used to measure the amount of quantum information conveyed in the noisy channel. We imagine that the initial state of system $Q$ is $\rho^{Q}$ which arises from the entanglement between $Q$ and a reference system $R$. Now the goal of Alice in $Q$ is to send the initial state $\rho^{Q}$ to Bob via a quantum channel (which can be described by $\varepsilon^{Q}$ ) and establish an entanglement between reference system $R$ and Bob's output system $Q^{\prime}$. The coherent information $I_{e}$ can express the degree of achieving this aim. In above investigation we just model the time evolution of quantum information transmitted via a noisy quantum channel described by the interaction with a control qubit. By comparing the coherent information $I_{e}$ and the concurrence, we find that $I_{e}$ is indeed a good measure for the capacity of a quantum channel to transmit the entanglement.

In summary, for an example modeling interaction with environment we derive the analytic expressions of the entropy exchange and the coherent information. From these we find that both the entropy exchange and the coherent information have profound correlations with the measure of the entanglement.

Acknowledgments This work was supported by the State Key Programs for Basic Research of China (Grants No. 2005CB623605 and No. 2006CB921803), and by National Foundation of Natural Science in China Grant No. 10474033 and No. 60676056.
[1] W.K. Wootters, Phys. Rev. Lett. 80, 2245(1998).

[2] C.H. Bennett, H.J. Bernstein, S. Popescu and Benjamin Schumacher, Phys. Rev. A 53, 2046(1996).

[3] S. Popescu and D. Rohrlich, Phys. Rev. A 56, R3319(1997).

[4] Thomas F. Jordan, Anil Shaji and E.C.G. Sudarshan, e-print arXiv:quant-ph/0704.0461v1(2007).

[5] Yang Xiang and Shi-Jie Xiong, e-print arXiv:quantph/0704.2973v3(2007)(to be published in Phys. Rev. A).
[6] Benjamin Schumacher and M.A. Nielsen, Phys. Rev. A 54, 2629(1996).

[7] Benjamin Schumacher, Phys. Rev. A 54, 2614(1996).

[8] K. Kraus, Ann. of Phys. (N.Y.) 64, 311(1971).

[9] W.F. Stinespring, Proc. Am. Math. Soc. 6, 211(1955).

[10] Howard Barnum, M.A. Nielsen and Benjamin Schumacher, Phys. Rev. A 57, 4153(1998).

[11] E. Schmidt, Ann. Math. 63, 433(1907). 